\title{
Glimepiride and Metformin Combinations in Diabetes Comorbidities and Complications: Real- World Evidence
}

\author{
Rakesh Kumar Sahay ${ }^{1}$, Vinod Mittal ${ }^{2}$, G Raja Gopal ${ }^{3}$, Sunil Kota ${ }^{4}$, Ghanshyam Goyal ${ }^{5}$, Mahesh \\ Abhyankar $^{6}$, Santosh Revenkar ${ }^{6}$ \\ 1. Department of Endocrinology, Osmania Medical College, Hyderabad, IND 2. Centre for Diabetes \& Metabolic \\ Diseases, Delhi Heart \& Lung Institute, New Delhi, IND 3. Department of Endocrinology and Diabetes, Sriridhi \\ Endocrinology \& Diabetes Super Specialty Clinic, Kurnool, IND 4. Department of Endocrinology, Endocare Hospital, \\ Vijaywada, IND 5. Department of Endocrinology, S K Diabetes Research and Education Centre, Kolkata, IND 6. \\ Scientific Services, USV Private Limited, Mumbai, IND
}

Corresponding author: Mahesh Abhyankar, drm.abhyankar@gmail.com

\section{Abstract \\ Objective}

To evaluate the usage of various strengths of glimepiride and metformin fixed-dose combinations in the management of type 2 diabetes mellitus (T2DM) patients with comorbidities and complications.

\section{Methods}

A retrospective, non-randomized, non-comparative, multi-centric real-world study included T2DM patients (age > 18 years) taking glimepiride and metformin fixed-dose combinations. Age, duration of diabetes, diabetes complications, comorbidities (hypertension and dyslipidemia), dosage frequency, and concomitant medications were analyzed from medical charts.

\section{Results}

A total of $4858 \mathrm{~T} 2 \mathrm{DM}$ patients were included, with a mean age of 52.67 years and males being predominant in the study population (60.85\%). The laboratory investigations showed a mean glycated hemoglobin (HbA1c) of 7.5, low-density lipoprotein (LDL) cholesterol of $104.81 \pm 38.19 \mathrm{mg} / \mathrm{dL}$, and serum creatinine of $0.88 \pm 0.26 \mathrm{mg} / \mathrm{dL}$. Around 2055 (42.30\%) T2DM patients were hypertensive, and telmisartan alone and a telmisartan-based combination were the drugs of choice for hypertension management. Similarly, 1073 (22.08\%) T2DM patients were having dyslipidemia and were primarily managed with rosuvastatin and its combination in 664 (62\%) patients. Macrovascular complications were observed in 339 (6.97\%) T2DM patients, among which coronary artery disease (CAD) had maximum prevalence, affecting 273 (5.61\%) T2DM patients. Microvascular complications were 1010 (20.79\%) T2DM patients, among which neuropathy had affected a maximum of 686 (14.12\%) followed by retinopathy (2.34\%) and nephropathy (1.81\%). Among the available 11 strengths, the glimepiride $2 \mathrm{mg}$ and metformin $500 \mathrm{mg}$ combination were most widely

Received 08/06/2020

Review began 08/25/2020 Review ended 09/26/2020 Published 09/28/2020

\section{(c) Copyright 2020}

Sahay et al. This is an open access article distributed under the terms of the Creative Commons Attribution License CC-BY 4.0., which permits unrestricted use, distribution, and reproduction in any medium, provided the original author and source are credited. prescribed in 1297 (26.69\%), followed by glimepiride $1 \mathrm{mg}$ and metformin $500 \mathrm{mg}$ in 1193 (24.57\%) patients, and the preferred dosage pattern was twice a daily in 2665 (54.85\%) T2DM patients. An age-wise prescription analysis showed that glimepiride and metformin combinations were the preferred choice for the management of diabetes across all the age groups.

\section{Conclusion}

The real-world evidence in the Indian clinical setting indicates that glimepiride and metformin fixed-dose combinations are widely used in the management in T2DM patients with comorbidities like hypertension, dyslipidemia, and diabetes complications. Glimepiride and metformin fixed-dose combinations are suitable for early as well as long-standing diabetes.

Categories: Endocrinology/Diabetes/Metabolism, Internal Medicine

Keywords: glimepiride, metformin, fixed dose combination, long standing, durability

\section{Introduction}

Diabetes mellitus has become a global burden, affecting 463 million people worldwide and responsible for 4.2 million deaths [1]. Type 2 diabetes mellitus (T2DM) is a heterogeneous disorder, and patients are at increased risk of developing macrovascular complications (coronary artery disease (CAD), peripheral arterial disease (PAD), and stroke/transient ischemic attack (TIA)) and microvascular complications (diabetic nephropathy, neuropathy, and retinopathy) due to inadequate glycemic control [2]. In India, the epidemic of diabetes continues to rise, which leads to disability and fatal complications that are related to an increased financial burden. According to the UK Prospective Diabetes (UKPDS) study, glycemic control using a single 
oral hypoglycemic agent is likely to be ineffective in the longer duration of disease, thus, unavoidably, many patients will require combination therapy to achieve their target glucose level [3]. The combination of modern sulfonylurea (glimepiride) and metformin is widely prescribed for effective blood glucose control due to its capability of counteracting "insulin secretion disorder" and "insulin resistance," respectively. In India, multiple strengths of the glimepiride and metformin fixed-dose combination are available and widely used by primary care physicians and specialists [4]. The current study is aimed to analyze the usage of various strengths of the glimepiride and metformin combination in the management of T2DM patients with comorbidities and complications.

\section{Materials And Methods}

A retrospective, non-randomized, non-comparative, multi-centric, real-world study of Glycomet Glimepiride (REAL GGP) was conducted in Indian health care centers having the medical records of adult T2DM patients (age $>18$ years) who had received treatment with any strengths of glimepiride and metformin SR (sustained-release) combination as per the standard of care in a physician's practice. The data collected were analyzed for age, diabetes complications (microvascular and macrovascular), comorbidities (hypertension and dyslipidemia), the dosage frequency and strengths of the glimepiride and metformin combination, and the durability of glycemic control across all age groups (>18 years).

The study was conducted in accordance with the ethical principles that are consistent with the Declaration of Helsinki, International Conference on Harmonization-Good Clinical Practices, and the applicable legislation on non-interventional studies. The current study is Clinical Trials Registry India (CTRI) registered (CTRI/2018/09/015693) and the study protocol was approved by an Independent Ethics Committee.

\section{Results}

\section{Population characteristics}

The study enrolled 4858 T2DM patients across India, i.e., 3030 patients from the south zone, 1239 patients from the east zone, 439 patients from the north zone, and 150 patients from the west zone. The mean age was $52.67 \pm 11.53$ years, with glycated hemoglobin (HbA1c) of $7.5 \%, 2956$ (60.85\%) patients were men, and mean weight was $71.07 \pm 11.59 \mathrm{~kg}$. The laboratory investigations showed mean total cholesterol of $180.21 \pm$ $42.36 \mathrm{mg} / \mathrm{dL}$, low-density lipoprotein (LDL) cholesterol of $104.81 \pm 38.19 \mathrm{mg} / \mathrm{dL}$, high-density lipoprotein (HDL) cholesterol of $45.58 \pm 18.34 \mathrm{mg} / \mathrm{dL}$, triglyceride of $164.13 \pm 81.74 \mathrm{mg} / \mathrm{dL}$, and serum creatinine of 0.88 $\pm 0.26 \mathrm{mg} / \mathrm{dL}$ (Table 1$)$. 


\section{Cureus}

Population Characteristics (n)

Age (years, mean $\pm S D$ )

$52.67 \pm 11.53$

Male, n (\%)

2956 (60.85\%)

Female, n (\%)

$1902(39.15 \%)$

Weight (kg, mean $\pm S D$ )

$71.07 \pm 11.59$

Laboratory Investigations

HbA1c

7.5

FPG $(\mathrm{mg} / \mathrm{dL})$

$147.53 \pm 48.99$

PPG (mg/dL)

$211.51 \pm 64.99$

Total Cholesterol (mg/dL)

$180.21 \pm 42.36$

$\operatorname{LDL}(\mathrm{mg} / \mathrm{dL})$

$104.81 \pm 38.19$

$\mathrm{HDL}(\mathrm{mg} / \mathrm{dL})$

$45.58 \pm 18.34$

Triglycerides (mg/dL)

$164.13 \pm 81.74$

Serum creatinine $(\mathrm{mg} / \mathrm{dL})$

$0.88 \pm 0.26$

Comorbidity

Hypertension

$2055(42.30 \%)$

Dyslipidemia

$1073(22.08 \%)$

Macrovascular complications [n (\%)]

Coronary artery disease

$276(5.66 \%)$

Stroke

$63(1.29 \%)$

Microvascular complications [n (\%)]

Neuropathy

$686(14.12 \%)$

Retinopathy

$114(2.34 \%)$

Nephropathy

$88(1.81 \%)$

Erectile dysfunction

$85(1.74 \%)$

Foot ulcer

$37(0.76 \%)$

Age-wise usage of glimepiride and metformin FDC [n (\%)]

18-40 years

$696(14.31 \%)$

40-60 years

$3798(78.18 \%)$

$>60$ years

$365(7.51 \%)$

TABLE 1: Baseline characteristic and laboratory investigations

Clinical usage of glimepiride and metformin fixed-dose combinations

Strengths of the Combination

Among the available 11 strengths, the glimepiride $2 \mathrm{mg}$ and metformin $500 \mathrm{mg}$ combination were most widely prescribed in 1297 (26.69\%) patients with T2DM, followed by glimepiride $1 \mathrm{mg}$ and metformin $500 \mathrm{mg}$ in 1193 (24.57\%), and glimepiride $0.5 \mathrm{mg}$ and metformin $500 \mathrm{mg}$ in 640 (13.17\%) patients with T2DM (Table 2). 


\section{Cureus}

\begin{tabular}{|c|c|c|}
\hline Sr. No. & Strengths of glimepiride and metformin combinations & Number of patients (\%) \\
\hline 1 & Glimepiride $0.5 \mathrm{mg}$ and Metformin $500 \mathrm{mg}$ & $640(13.17 \%)$ \\
\hline 2 & Glimepiride $0.5 \mathrm{mg}$ and Metformin $1000 \mathrm{mg}$ & $175(3.6 \%)$ \\
\hline 3 & Glimepiride $1 \mathrm{mg}$ and Metformin $500 \mathrm{mg}$ & $1194(24.57 \%)$ \\
\hline 4 & Glimepiride $1 \mathrm{mg}$ and Metformin $850 \mathrm{mg}$ & $72(1.48 \%)$ \\
\hline 5 & Glimepiride $1 \mathrm{mg}$ and Metformin $1000 \mathrm{mg}$ & $393(8.08 \%)$ \\
\hline 6 & Glimepiride $2 \mathrm{mg}$ and Metformin $500 \mathrm{mg}$ & $1297(26.69 \%)$ \\
\hline 7 & Glimepiride $2 \mathrm{mg}$ and Metformin $1000 \mathrm{mg}$ & $528(10.86 \%)$ \\
\hline 8 & Glimepiride $2 \mathrm{mg}$ and Metformin $850 \mathrm{mg}$ & $232(4.76 \%)$ \\
\hline 10 & Glimepiride $3 \mathrm{mg}$ and Metformin $1000 \mathrm{mg}$ & $200(4.11 \%)$ \\
\hline 11 & Glimepiride $4 \mathrm{mg}$ and Metformin $1000 \mathrm{mg}$ & $127(2.61 \%)$ \\
\hline
\end{tabular}

TABLE 2: Usage of the various strengths of glimepiride and metformin combinations

Dosage Pattern

The prescription of glimepiride and metformin dosage was individualized according to the patients, among which BID (twice a day) was the preferred dosing frequency in 2665 (54.85\%) patients with T2DM while OD (once a day) was prescribed in 2193 (45.14\%) patients with T2DM (Figure 1).

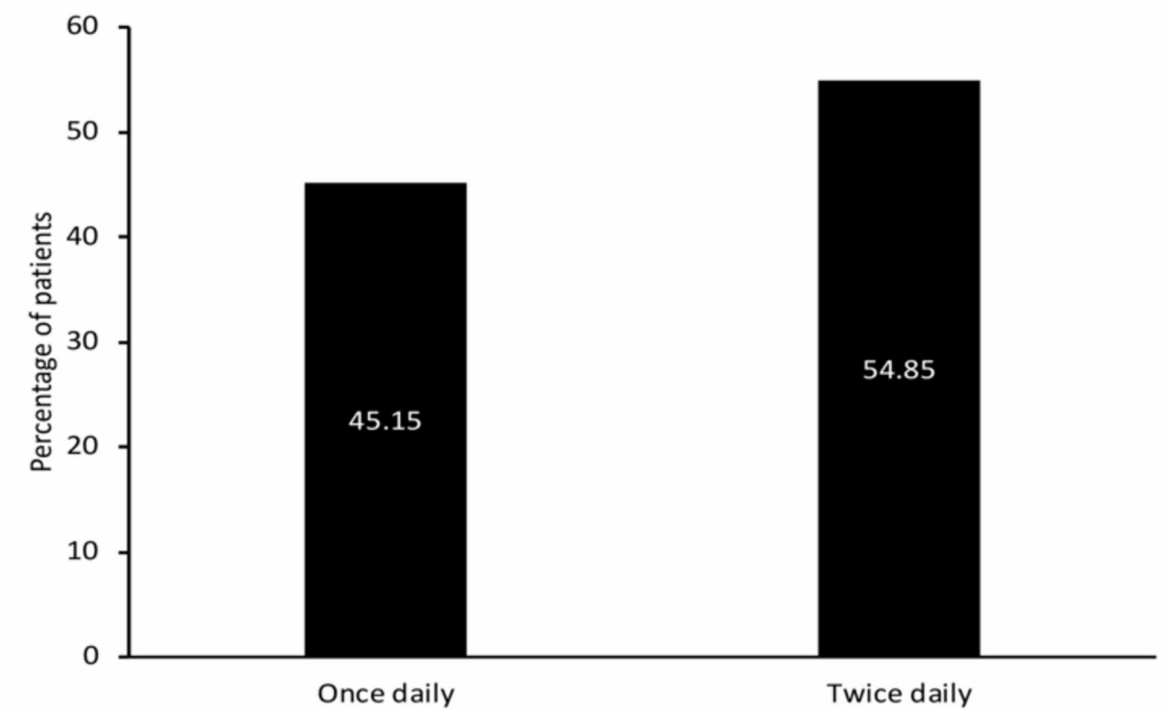

FIGURE 1: Dose frequency of glimepiride and metformin fixed-dose combinations

\section{Durability of the glimepiride and metformin combination}

Around 3798 patients with T2DM (78.18\%) on the glimepiride and metformin combination were in the age group of $40-60$ years, followed by $14.3 \%$ patients of $<40$ years of age, and 365 patients ( $7.15 \%$ ) patients $>60$ years of age (Table 1). The duration of diabetes in the study population ranged from two years to 12 years across the age groups; this highlights the glycemic durability of the glimepiride and metformin combination (Figure 2). The mean duration of diabetes was 8.8 years, 11.5 years, and 12.9 years in patients of $60-70$ years of age, $70-80$ years of age, and over 80 years of age, respectively. All the strengths of glimepiride and metformin are used for T2DM patients $>60$ years of age and having long-standing diabetes. 


\section{Cureus}

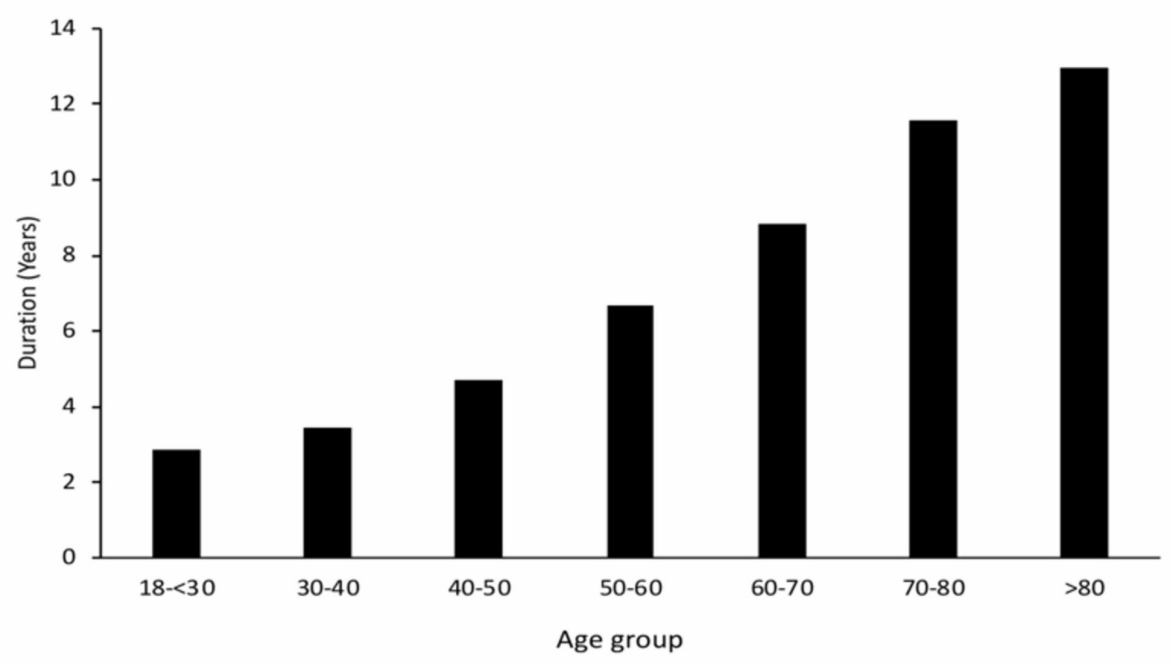

FIGURE 2: Duration of diabetes in patients taking glimepiride and metformin

\section{Diabetes comorbidities}

Hypertension

In total, 2055 (42.30\%) patients with T2DM were having hypertension as comorbidity (Table 1). Telmisartan alone and telmisartan-based combinations were the most common antihypertensive therapy used in 1322 (64\%) patients, followed by olmesartan and its combination in 301 (11\%) patients (Figure 3).

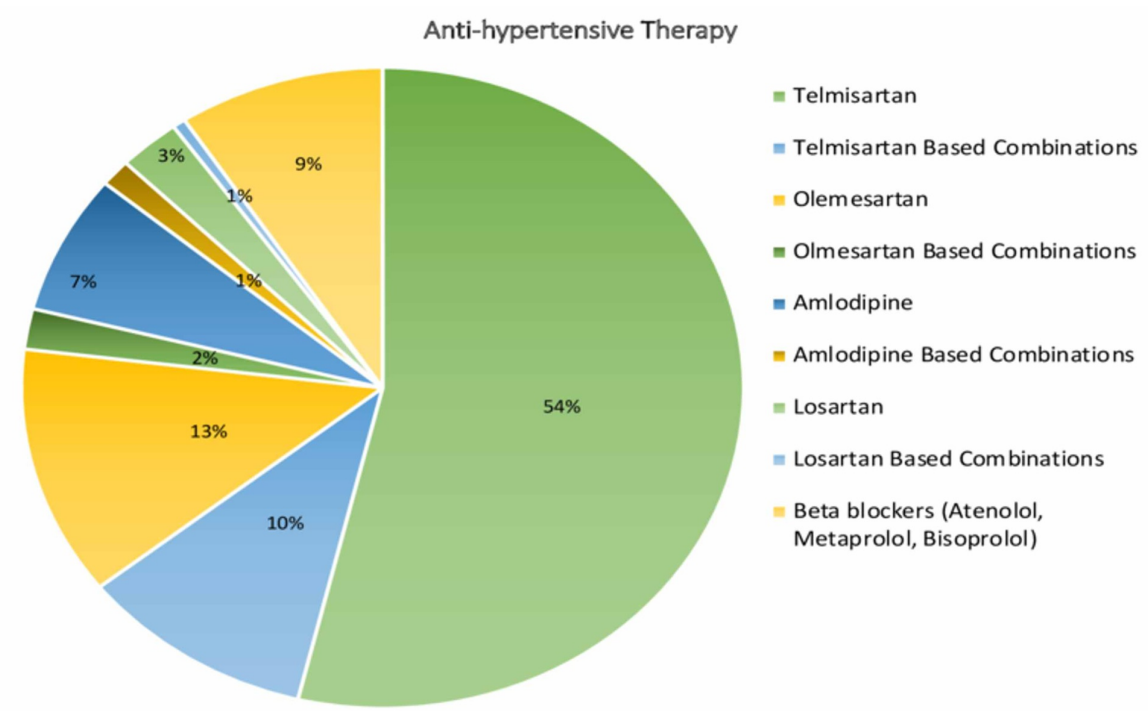

FIGURE 3: Anti-hypertensive therapy in hypertension with T2DM

T2DM: type 2 diabetes mellitus

Dyslipidemia

Dyslipidemia was observed in 1073 (22.08\%) patients (Table 1). The study result showed that rosuvastatin and its combination was the choice of lipid-lowering therapy in 664 (62\%) of patients, followed by atorvastatin and its combination in 391 (36\%) patients with T2DM (Figure 4). 


\section{Cureus}

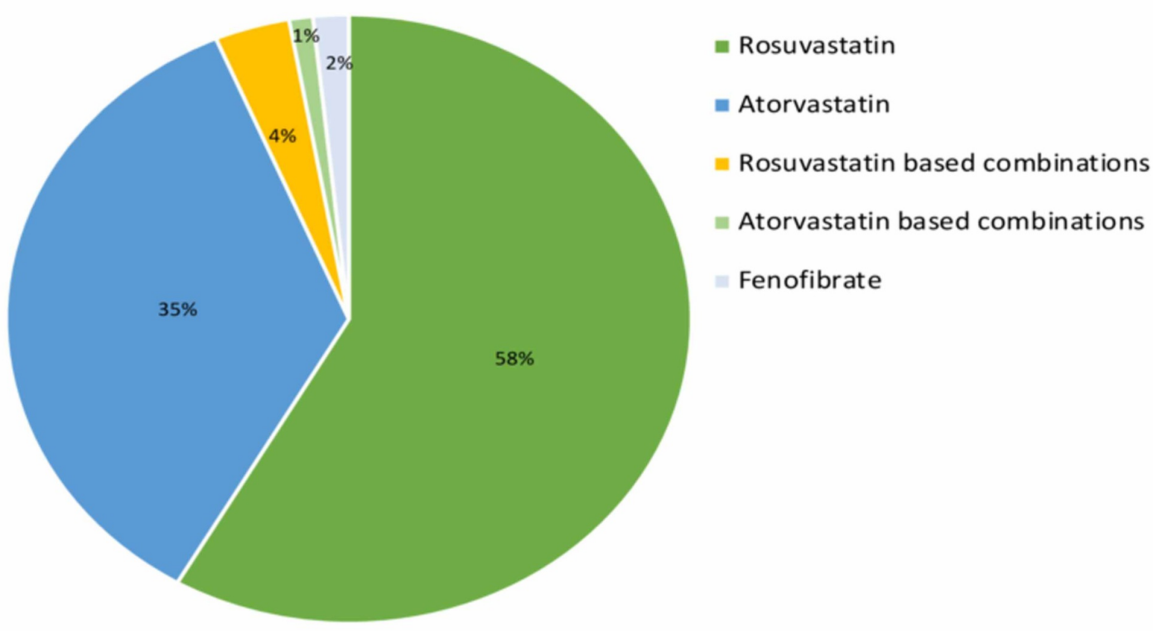

FIGURE 4: Lipid-lowering therapy in dyslipidemia with T2DM

T2DM: type 2 diabetes mellitus

\section{Diabetes complications}

Macrovascular Complications

The results showed that 339 (6.97\%) T2DM patients had macrovascular complications, among which coronary artery disease (CAD) was present in 276 (5.68\%) T2DM patients, followed by stroke in 63 (1.29\%) patients (Table 1). As primary or secondary prevention, aspirin was administered as monotherapy or dual antiplatelet therapy (DAPT) in 7\% of T2DM patients.

Microvascular Complications

Among the patients with T2DM, 1010 (20.79\%) had microvascular complications. Neuropathy was the leading microvascular complication affecting 686 (14.12\%) T2DM patients and the preferred choice of treatment was pregabalin and its combination. Retinopathy was observed in 114 (2.34\%), nephropathy in 88 (1.81\%), erectile dysfunction in 85 (1.74\%), and foot ulcer in 37 (0.76\%) T2DM patients.

\section{Discussion}

All the strengths of the glimepiride and metformin combination are widely prescribed by the physicians across India, considering the efficacy and cost-effectiveness of the combination therapy [5].

The present real-world study describes the usage of various strengths of the glimepiride and metformin combination in patients with T2DM having hypertension and dyslipidemia as the major comorbidities and showing the presence of micro- and macrovascular complications.

The results of the present study indicate good glycemic control at all the age groups and showed a sustained legacy effect maintained for a longer duration of diabetes, which was in concordance with the study conducted by Kalra S et al. [4]. Early combination therapy with the glimepiride and metformin combination could provide the benefit of a legacy effect through earlier glucose control, avoiding a negative glycemic memory related to micro- and macrovascular complications [6]. Hypertension is a major risk factor for cardiovascular disease (CVD) [7], and the presence of hypertension in diabetes patients increases the risks of micro- and macrovascular complications and mortality [1].

In the present study, hypertension was the most common comorbidity observed, which highlights the fact that at least one antihypertensive drug should be administered to patients with diabetes to reduce cardiovascular (CV) morbidity and mortality [7]. As per Derosa G et al. antihyperglycemic drugs might have a small but clinically significant effect on blood pressure in patients with T2DM [8]. Despite the various antidiabetic agents, the glimepiride and metformin combination was the preferred choice in hypertensive patients with diabetes for optimal blood glucose control, which was in concordance with the previous study by Tripathi $\mathrm{S}$ et al [5]. The hypertension treatment regimen in the current study showed that telmisartan and telmisartan-based combinations were the preferred choices of antihypertensive therapy in $64 \%$ of patients, followed by olmesartan and its combination in $11 \%$ of patients. These results were in concordance with Guerrero-García C et al. who stated that in hypertensive diabetes patients, multiple drug therapy is 
necessary to achieve blood pressure targets [9]. Studies report the CV protection of metformin, as it reduces the risk factors of CV diseases, including lipids, overweight, and hypertension; thus, lowering the incidence of CVD [10-11]. The conventional sulfonylureas are reported to have adverse CV effects while studies report glimepiride to have neutral CV effects [12]. A preliminary study of glimepiride conducted in 34 patients with T2DM showed positive effects on biomarkers related to CV regulation, indicating the presence of antioxidant, anti-inflammatory, and angiogenic properties [13].

Patients with T2DM have an increased prevalence of lipid abnormalities and, consequently, a high risk of atherosclerotic cardiovascular disease (ASCVD). Increased levels of LDL cholesterol and decreased levels of HDL cholesterol are the strongest predictors of CAD in patients with T2DM [14]. In the current study, dyslipidemia was observed in $22.08 \%$ of patients, which highlights the importance of lipid management as an integral part of T2DM management. Glimepiride does not show significant effects on lipid profile [15] while metformin is found to improve dyslipidemia in patients with T2DM by reducing LDL, triglycerides, and increasing HDL-C levels [16]. Kashi et al. [17] have reported a synergistic effect between metformin and statin, which is beneficial to reduce CV events in high-risk patients.

Macrovascular complications in diabetes primarily affect coronary arteries, peripheral arteries, and cerebrovasculature. Early macrovascular disease is associated with atherosclerotic plaque in the vasculature supplying blood to the heart, brain, limbs, and other organs while a late stage involves complete blockage of these vessels, which can increase the risks of myocardial infarction (MI), stroke, claudication, and gangrene [18]. Hence, the main target in the management of T2DM is maintaining good glycemic control, which is particularly important for controlling diabetes and preventing and delaying diabetes complications [19]. The CAROLINA (Cardiovascular Outcome Study of Linagliptin vs Glimepiride in Type 2 Diabetes) trial conducted in patients with T2DM at an elevated cardiovascular risk demonstrated that glimepiride is at par with linagliptin for safety from a three-point major cardiovascular event (3P-MACE) and 4P-MACE. Similarly, the South Asian Federation of Endocrine Societies (SAFES) consensus statement recommended that modern sulfonylureas (e.g. glimepiride) should be preferred over conventional sulfonylureas given the reduced mortality (all-cause and CV mortality) and better CV outcomes [20].

Micro-vessels are the basic functional unit of the CV system. They also regulate vascular permeability and myogenic responses, which can adapt blood flow according to local metabolic needs [18]. Diabetes induces pathognomonic changes in the microvasculature, affecting the capillary basement membrane, including arterioles in the glomeruli, retina, myocardium, skin, and muscle, by increasing their thickness, leading to the development of diabetic microangiopathy [21]. Hence, optimal glycemic control and achieving targeted blood glucose levels is important for the prevention of microvascular complications [22]. In the current study, 20.79\% of patients with T2DM had microvascular complications, of which neuropathy was the leading microvascular complication affecting patients with T2DM and preferably treated with pregabalin and its combination. The UKPDS (United Kingdom Prospective Diabetes Study) showed that early intensive glucose control at the time of diagnosis is associated with a significant decrease in the risk of myocardial infarction and death from any cause, along with a reduction in the risk of microvascular disease [23], thus indicating the important role of glycemic control to reduce microvascular complications.

According to the American Diabetes Association (ADA) and the Research Society for Study of Diabetes in India (RSSDI) wheel, modern sulfonylureas, such as glimepiride have high efficacy over conventional sulfonylureas and lower risk of hypoglycemia with neutral CV risk [24-25].

A case-based questionnaire conducted with 2248 Indian patients showed that multiple strengths of glimepiride and metformin combinations are prescribed in T2DM patients, irrespective of age, diabetes duration, body mass index (BMI), and diabetes complications [26]. These combinations have shown good clinical efficacy, tolerability, and glycemic control and are beneficial particularly in resource constraint locations. The clinicians are benefited from these combinations due to the ease of up- and down-titration.

Glimepiride in combination with metformin is not only safe and effective but also cost-effective and an easily accessible approach for the management of T2DM [27]. A pharmacoeconomic study showed that the glimepiride and metformin combination was more cost-effective than the metformin and teneligliptin combination [28]. Glimepiride is comparable to other newer agents, such as DPP4i and GLP-1, in achieving the glycemic goal but at a low cost [29]; correspondingly, the results of reported studies confirm the costeffectiveness of metformin over other anti-diabetic agents and lifestyle interventions [30]. These combinations are available widely in various strengths and easily accessible at affordable prices.

One of the key limitations of this study was the retrospective collection of data, which limited the strength of inference that can be made from the observations. The collection of data did not include fasting and the postprandial blood glucose levels and BMI of the patients.

\section{Conclusions}

The real-world analysis concludes that all the strengths of the glimepiride and metformin combinations are widely prescribed in diabetes with comorbidities like hypertension and dyslipidemia and complications for 
optimal glycemic control. Glimepiride and metformin fixed-dose combinations are suitable for early as well as long-standing diabetes.

\section{Additional Information \\ Disclosures}

Human subjects: Consent was obtained by all participants in this study. Clinicon Independent Ethics Committee for evaluation of protocols for clinical research issued approval 07/08/2018. Approved. As this was a retrospective study consent of participants was not necessary. Animal subjects: All authors have confirmed that this study did not involve animal subjects or tissue. Conflicts of interest: In compliance with the ICMJE uniform disclosure form, all authors declare the following: Payment/services info: This project has been funded by USV Pvt Ltd. Mumbai. Financial relationships: Dr. Mahesh Abhyankar and Dr. Santosh Revankar declare(s) employment from USV Pvt Ltd., Mumbai. Both authors are employees of USV Pvt Ltd., Mumbai. Other relationships: All authors have declared that there are no other relationships or activities that could appear to have influenced the submitted work.

\section{Acknowledgements}

We acknowledge Ms. Farida Hussain, Mr. A Thamburaj, and Ms. Shashikala Borhade from USV Pvt. Ltd. for their assistance in the conduct of the project. Medical writing support was provided by Dr. Rohit Malabade from the scientific services of USV Pvt. Ltd. We acknowledge Abiogenesis Clinpharm Pvt. Ltd. for their services in the conduction of the real-world study. Contributors: Dr. A C Shyam, Dr. A Jeswanth, Dr. A Praveen Kumar Reddy, Dr. A Ravi Kumar, Dr. Ajay Patwari, Dr. Amrit Nanaiah, Dr. M Mallikarjuna Rao, Dr. Ankur Sinha, Dr. Anuj Kumar Saha, Dr. Arun Karthik K, Dr. Arundhati Dasgupta, Dr. Ashok Kumar, Dr. B Karthik Rao, Dr. B R K Prakash, Dr. B Rajendra Kumar, Dr. B Ravi Kumar, Dr. Balaram Panigrahi, Dr. Bensley L Gonsalves, Dr. Bharat Panigrahi, Dr. Bhuvana Priya, Dr. Biswajit Mukherjee, Dr. Brij Kishore Prasad, Dr. C H Naveen Kumar, Dr. C Mallikarjun, Dr. C Pradeep, Dr. C V N Kavitha Rao, Dr. Chakkravarthi D, Dr. Chandrakanta Mishra, Dr. D Mathru, Dr. D Narayana Reddy, Dr. Durga Kumar Srivastav, Dr. Durga Prasad Chakraborty, Dr. G K Mahesh, Dr. G Ramakrishna Reddy, Dr. G V Siva Reddy, Dr. G Kiran, Dr. Girithara Gopalakrishnan, Dr. Gopal Agarwal, Dr. Govindappa.D, Dr. Guruprasad U, Dr. H S Karunesh Kumar, Dr. Harish Chandra Mishra, Dr. J Senthil Kumar, Dr. Jadumani Nayak, Dr. Jagdish Mulchandani, Dr. Jagdish Singh, Dr. Jishnu Banerjee, Dr. K Babu Chakkaravarthy, Dr. K Balasubramanian Dr. K M Jeyabalaji, Dr. K Vamsi Krishna, Dr. Katakam Lavanya, Dr. M G Uvaraj, Dr. M Leelavathi, Dr. M Mallikarjuna Rao, Dr. M Prabhurami Reddy, Dr. M R Vidhya, Dr. M Sreekanth Reddy, Dr. Madhusudhan Reddy K, Dr. Manoj Kumar Dash, Dr. Mohammed Idrees, Dr. Mohammed Ishaq Ahmed, Dr. N Kotresh, Dr. N Sudha, Dr. Nishad S K, Dr. P Chakradhar, Dr. P Chandramohan, Dr. P Hari Kumar Reddy, Dr. P Sateesh Kumar Raju, Dr. P Sudhakar Reddy, Dr. Pankaj M Patel, Dr. Paramesh S, Dr. Paresh Kumar Jena, Dr. Pradeep Sehgal, Dr. Pradip Gupta, Dr. Prasad G M, Dr. Prasanna Belgundi, Dr. Pratap Kumar Mishra, Dr. Praveen Gupta, Dr. R Shashi Kumar, Dr. R Sivagnanam, Dr. R Thirumurugan, Dr. Rabindra Kumar Das, Dr. Raj Mohan L, Dr. Rajeev Bansal, Dr. Rajesh Gupta, Dr. Rajesh Kappala, Dr. Rajkumar M, Dr. Rakesh Prasad, Dr. Ramesh Kumar Goenka, Dr. Ravi Kumar Y S, Dr. Ritesh Kumar Agrawala, Dr. S Naga Narasimhaiah, Dr. S Nallamuthusamy, Dr. S P Rajesh Dhilip Sydney, Dr. S P Sathish Kumar, Dr. S Satyanarayana Murthy, Dr. Saibal Chakravorty, Dr. Samrat D Shah, Dr. Sanjay Kumar Jain, Dr. Sanjay Teotia, Dr. Sanjiv Zutshi, Dr. Shreekant Heramb Dike, Dr. Sidhartha Dash, Dr. Sriprasad Mohanty, Dr. Sudhir Patnaik, Dr. Sumit Kumar Chakraborty, Dr. Surendra Kumar Bhatter, Dr. Suresh Chandra Sahoo, Dr. Tanmoy Mandal, Dr. Umakanta Mishra, Dr. Umarani R, Dr. Utkal Kishore Khadanga, Dr. V V Satya Sekhar, Dr. Vijith Kumar K. Authorship: All named authors take the responsibility for the integrity of the work as a whole and have given their approval for this version to be published. The study was conducted under ethical guidelines. The contents published herein represent the views and opinions of the various contributing authors and does not necessarily represent the views or opinion of USV Pvt Ltd and/or its affiliates. The details published herein are intended for the dissemination of information for educational, academic, and/or research purposes and are not intended as a substitute for professional medical advice, diagnosis, or treatment.

\section{References}

1. IDF diabetes atlas. 9th edition. (2019). https://diabetesatlas.org/en/.

2. American Diabetes Association: Microvascular complications and foot care: standards of medical care in diabetes-2019. Diabetes Care. 2019, 42:124-138. 10.2337/dc19-S011

3. Chawla A, Chawla R, Jaggi S: Microvascular and macrovascular complications in diabetes mellitus: Distinct or continuum?. Indian J Endocr Metab. 2016, 20:546-551. 10.4103/2230-8210.183480

4. Kalra S, Das AK, Baruah MP, et al.: Glucocrinology of modern sulfonylureas: Clinical evidence and practicebased opinion from an international expert group. Diabetes Ther. 2019, 10:1577-1593. 10.1007/s13300-0190651-1

5. Tripathi S, Tiwaskar M, Kota S, Parthan G, Dasgupta A, Mohanasundaram S, Mohan V: Need of single pill fixed-dose combination with glimepiride in management of diabetes mellitus. J Assoc Physicians India. 2019, S1:30-33.

6. Kim HS, Kim DM, Cha BS, et al.: Efficacy of glimepiride/metformin fixed-dose combination vs metformin uptitration in type 2 diabetic patients inadequately controlled on low-dose metformin monotherapy: a randomized, open label, parallel group, multicenter study in Korea. J Diabetes Investig. 2014, 5:701-708. 10.111/jdi.12201 
7. Passarella P, Kiseleva TA, Valeeva FV, Gosmanov AR: Hypertension management in diabetes: 2018 update. Diabetes Spectr. 2018, 31:218-224. 10.2337/ds17-0085

8. Derosa G, Sibilla S: Optimizing combination treatment in the management of type 2 diabetes . Vasc Health Risk Manag. 2007, 3:665.

9. Guerrero-García C, Rubio-Guerra AF: Combination therapy in the treatment of hypertension . Drugs Context. 2018, 7:212531. 10.7573/dic.212531

10. Loi H, Boal F, Tronchere H, et al.: Metformin protects the heart against hypertrophic and apoptotic remodeling after myocardial infarction. Front Pharmacol. 2019, 10:154-30873028. 10.3389/fphar.2019.00154

11. Charytan DM, Solomon SD, Ivanovich P, et al.: Metformin use and cardiovascular events in patients with type 2 diabetes and chronic kidney disease. Diabetes Obes Metab. 2019, 21:1199-1208. 10.1111/dom.13642

12. Geisen K, Végh A, Krause E, Papp JC: Cardiovascular effects of conventional sulfonylureas and glimepiride Horm Metab Res. 1996, 28:496-507. 10.1055/s-2007-979841

13. Nakamura I, Oyama J, Komoda H, et al.: Possible effects of glimepiride beyond glycemic control in patients with type 2 diabetes: a preliminary report. Cardiovasc Diabetol. 2014, 13:15. 10.1186/1475-2840-13-15

14. American Diabetes Association: Cardiovascular disease and risk management: standards of medical care in diabetes2125312019. Diabetes Care. 2019, 42:103-123. 10.2337/dc19-S010

15. Rosenblit PD: Common medications used by patients with type 2 diabetes mellitus: what are their effects on the lipid profile?. Cardiovasc Diabetol. 2016, 15:95. 10.1186/s12933-016-0412-7

16. Lin SH, Cheng PC, Tu ST, Hsu SR, Cheng YC, Liu YH: Effect of metformin monotherapy on serum lipid profile in statin-naïve individuals with newly diagnosed type 2 diabetes mellitus: a cohort study. PeerJ. 2018;6, 4578-29666753. 10.7717/peerj.4578

17. Kashi Z, Mahrooz A, Kianmehr A, Alizadeh A: The role of metformin response in lipid metabolism in patients with recent-onset type 2 diabetes: $\mathrm{HbA1c}$ level as a criterion for designating patients as responders or nonresponders to metformin. PLoS One. 2016, 11:0151543. 10.1371/journal.pone.0151543

18. Rahelić D, Javor E, Lucijanić T, Skelin M: Effects of antidiabetic drugs on the incidence of macrovascular complications and mortality in type 2 diabetes mellitus: a new perspective on sodium-glucose cotransporter 2 inhibitors. Ann Med. 2017, 49:51-62. 10.1080/07853890.2016.1226514

19. Kant R, Munir KM, Kaur A, Verma V: Prevention of macrovascular complications in patients with type 2 diabetes mellitus: review of cardiovascular safety and efficacy of newer diabetes medications. World J Diabetes. 2019, 10:324-332. 10.4239/wjd.v10.i6.324

20. Kalra S, Aamir AH, Raza A, et al.: Place of sulfonylureas in the management of type 2 diabetes mellitus in South Asia: a consensus statement. Indian J Endocr Metab. 2015, 19:577-596. 10.4103/2230-8210.163171

21. Almourani R, Chinnakotla B, Patel R, Kurukulasuriya LR, Sowers J: Diabetes and cardiovascular disease: an update. Curr Diab Rep. 2019, 19:161-174. 10.1007/s11892-019-1239-x

22. Aguiar C, Duarte R, Carvalho D: Nova abordagem para o tratamento da diabetes: da glicemia à doença cardiovascular. New approach to diabetes care: from blood glucose to cardiovascular disease [Article in Portuguese, English]. Rev Port Cardiol. 2019, 38:53-63. 10.1016/j.repc.2018.03.013

23. Stratton IM, Adler AI, Neil HA, et al.: Association of glycaemia with macrovascular and microvascular complications of type 2 diabetes (UKPDS 35): prospective observational study. BMJ. 2000, 321:405. 10.1136/bmj.321.7258.405

24. Riddle MC: A verdict for glimepiride: effective and not guilty of cardiovascular harm . Diabetes Care. 2019, 42:2161-2163. 10.2337/dci19-0034

25. Chawla R: RSSDI Diabetes Update 2018. JP Medical Ltd., New Delhi; 2018.

26. Unnikrishnan Ag, Pandit K, George J: Clinical utilization pattern of multiple strengths of glimepiride and metformin fixed dose combinations in Indian type 2 diabetes patients. J Assoc Physicians India. 2020, 68:5761.

27. Singh SK: Commentary on "consensus recommendations on sulfonylurea and sulfonylurea combinations in the management of type 2 diabetes mellitus: International Task Force". Indian J Endocrinol Metab. 2018, 22:158-159. 10.4103/ijem.IJEM_22_18

28. Tandon T, Dubey AK, Srivastava S, Manocha S, Arora E, Hasan N: A pharmacoeconomic analysis to compare cost-effectiveness of metformin plus teneligliptin with metformin plus glimepiride in patients of type-2 diabetes mellitus. J Family Med Prim Care. 2019, 8:955-959. 10.4103/jfmpc.jfmpc_22_19

29. Zhang Y, McCoy RG, Mason JE, Smith SA, Shah ND, Denton BT: Second-line agents for glycemic control for type 2 diabetes: are newer agents better?. Diabetes Care. 2014, 37:1338-1345. 10.2337/dc13-1901

30. Gu S, Tang Z, Shi L, Sawhney M, Hu H, Dong H: Cost-minimization analysis of metformin and acarbose in treatment of type 2 diabetes. Value Health Reg Issues. 2015, 6:84-88. 10.1016/j.vhri.2015.03.012 